\title{
LOUIS-P. BETZ.
}

\section{LA LITTÉRATURE COMPARÉE}

\section{ESSAI BIBLIOGRAPHIQUE}

\author{
INTRODUCTION \\ par \\ JOSEPH TEXTE. \\ DEUXIÈME ÉDITION AUGMENTÉE, \\ PUBLIÉE, AVEC UN INDEX MÉTHODIQUE, \\ par \\ FERNAND BALDENSPERGER, \\ Professeur à l'Université de Lyon.
}

STRASBOURG

KARL J. TRÜBNER, ÉDITEUR

I904. 
M. DuMont Schauberg, Strasbourg. 


\section{A MA CHERE FEMME.}

En souvenir de nos heures de travail commun à la Bibliothèque Nationale de Paris.

LOUIS-P. BETZ. 

.... Lasst alle Völker unter gleichem Himmel

Sich gleicher Gabe wohlgemuth erfreun.

Goethe. 

Ma religion littéraire et politique, c'est l'unité des

lettres et la fraternité des peuples modernes.

Edgar Quinet.

......Ueberall hört und liest man von dem Vorschreiten des Menschengeschlechts, von den weitern Aussichten der Welt- und Menschenverhälnisse. Wie es auch im Ganzen hiermit beschaffen sein mag, welches $z u$ untersuchen und näher zu bestimmen nicht meines Amtes ist, will ich doch von meiner. Seite meine Freunde aufmerksam machen, dass ich überzeugt sei, es bilde sich eine allgemeine Weltliteratur, worin uns Deutschen eine ehrenvolle Rolle vorbehalten ist.....

Eine jede Literatur ennuyirt sich zuletzt in sich selbst, wenn sie nicht durch fremde Theilnahme wieder aufgefrischt ist.

Goethe.

Tout peuple sans commerce intellectuel avec les autres peuples n'est qu'une maille rompue du grand filet.

Philarite Chasles.

C'est prouver sa jeunesse et sa force, c'est s'assurer un avenir de renouvellement et d'action au dehors, que de faire connaitre et de comprendre tont ce qui se fait de grand, de beau, de neuf en dehors de ses frontières, de s'en servir, sans l'imiter, de l'assimiler, de le transformer suivant sa nature propre, de conserver sa personnalité en l'élargissant et d'être ainsi toujours la même et toujours changeante, toujours nationale et toujour's européenne.

Gaston Paris.

Die vergleichende Literaturbetrachtung hat die doppelte Eigenschaft, uns das Fremde solchergestalt zu nähern, dass wir es uns aneignen können, und uns von dem Eigenen solchergestalt zu entfernen, dass wir es zu überschauen vermögen. Man sieht weder, was dem Auge allzu nahe, noch was demselben allzu fern liegt.

\section{G. Brandes.}

Rester soi-même et cependant s'unir aux autres, tel est le problème que chaque homme a à résoudve. Tel est le problème aussi de l'alliance des peuples de l'occident.

Saint-Marc Girardin.

..... La littérature nationale n'a jamais suffi, et aujourd'hui moins que jamais, à exprimer les sentiments dominants de notre société.

E. Hennequin.

Literature draws its sap from the deep soil of human nature's common and everlasting sympathies.

Lowell. 
